\title{
Tandem histone-binding domains enhance the activity of a synthetic chromatin effector
}

Stefan J. Tekel ${ }^{1}$, Daniel Vargas ${ }^{1}$, Lusheng Song ${ }^{2}$, Joshua LaBaer ${ }^{2}$, and Karmella A. Haynes ${ }^{1}$

1. School of Biological and Health Systems Engineering, Arizona State University, Tempe, AZ

2. Biodesign Institute, Arizona State University, Tempe, $A Z$

Keywords: chromatin, histones, transcription factor, protein engineering, epigenetics

Corresponding author:

Karmella A. Haynes

karmella.haynes@asu.edu

\begin{abstract}
Fusion proteins that specifically interact with biochemical marks on chromosomes represent a new class of synthetic transcriptional regulators that decode cell state information rather than DNA sequences. In multicellular organisms, information relevant to cell state, tissue identity, and oncogenesis is often encoded as biochemical modifications of histones, which are bound to DNA in eukaryotic nuclei and regulate gene expression states. We have previously reported the development and validation of the "Polycomb-based transcription factor" (PcTF), a fusion protein that recognizes histone modifications through a protein-protein interaction between its polycomb chromodomain (PCD) motif and trimethylated lysine 27 of histone H3 (H3K27me3) at genomic sites. We demonstrated that PcTF activates genes at methyl-histone-enriched loci in cancer-derived cell lines. However, PcTF induces modest activation of a methyl-histone associated reporter compared to a DNA-binding activator. Therefore, we modified PcTF to enhance its target affinity. Here, we demonstrate the activity of a modified regulator called $\mathrm{Pc}_{2} \mathrm{TF}$, which has two tandem copies of the H3K27me3-binding PCD at the N-terminus. $\mathrm{Pc}_{2}$ TF shows higher affinity for H3K27me3 in vitro and shows enhanced gene activation in HEK293 cells compared to PcTF. These results provide compelling evidence that the intrinsic histone-binding activity of the PCD motif can be used to tune the activity of synthetic histone-binding transcriptional regulators.
\end{abstract}




\section{INTRODUCTION}

The discovery of histone post-translational modifications (PTMs) and the peptides that specifically interact with these marks has enabled scientists and cell engineers to manipulate chromatin, the DNA-protein structure that regulates gene expression states in eukaryotic cells. Structure-based models have informed targeted knock-down of chromatin subunits and the rational design of low molecular weight inhibitor compounds (reviewed in [1]). DNA-binding domains fused with structural chromatin proteins and histone-modifying enzymes have been used to generate ectopic chromatin conformations at specific loci [2,3]. Until recently, scientists have not yet leveraged PTM-binding peptides from natural effector proteins to "read" the rich biological information encoded in histone marks in living cells. Peptides that recognize specific histone PTM signals are an essential requirement for synthetic systems that integrate epigenetic regulatory signals. In order to use PTM-binding peptides in synthetic fusion proteins, the peptides must be portable, that is, maintain their intrinsic function within a new protein sequence. Early studies established important foundational knowledge by demonstrating that the interaction of the chromodomain motif (CD) with trimethylated histone $\mathrm{H} 3$ lysine 27 (H3K27me3) is an intrinsic activity that is maintained by the CD in the context of recombinant, fusion proteins [4,5]. Other protein folds including the bromodomain (BRD) and plant homeodomain finger (PHD) function as isolated peptides [6-8] and within fusion proteins $[7,9]$ to specifically interact with acetylated histone lysines (BRD) and H3K4me3 (PHD).

We constructed the Polycomb-based Transcription factor (PcTF) using a histone PTM-binding motif from the natural protein CBX8 [10]. The CBX8 effector protein binds to histone H3 trimethylated at lysine 27 (H3K27me3) through its N-terminal Polycomb chromodomain (PCD) and establishes a silenced transcriptional state. Expression of PcTF, an artificial transcriptional activator with an N-terminal PCD, mCherry tag, and C-terminal VP64 activation domain, led to increased expression of H3K27me3-enriched genes in three different cancer-derived cell lines $[10,11]$. These results show promise for designing transcription factors that can read chromatin marks to rewire aberrant epigenetic programming. However, binding affinities observed in vitro for isolated PCD domains is poor, reported as 5 - $165 \mu \mathrm{M}[12,13]$, compared to DNA-binding domains with target affinities in the pico- to nanomolar range such as TALEs ( 3 - $220 \mathrm{nM})$, [14], Zinc Fingers ( 0.01 - $16 \mathrm{nM})[15,16]$, and CRISPR/Cas ( 0.5 nM) [17]. In other work, we observed stronger gene upregulation when mCherry-VP64 was targeted to a promoter via a Gal4 DNA binding domain compared to the PCD histone binding domain [18]. PcTF-mediated gene activation is dose-dependent [11], and high PcTF expression levels are required for optimal activity. This limits the usefulness of PcTF for therapeutic applications where barriers to delivery severely limits 
the number of proteins that ultimately reach the nuclei of target cells. Although pharmacokinetic barriers to DNA and protein delivery in vivo are not trivial, increasing the intracellular performance of PcTF is a critical step for advancing this technology towards clinical use.

Here, we extend our investigation of the utility of the PCD motif as a protein design tool by testing its ability to confer multivalency to a synthetic regulator. In this report, multivalency is defined as the engagement of a single protein (monomer) with more than one histone PTM (reviewed in $[1,3,19]$ ). Multivalent chromatin proteins can engage adjacent PTMs within a single histone tail, such as K4me3 and R8me2 on histone H3 bound by Spindlin1 [20], or K5ac and $\mathrm{K} 12 \mathrm{ac}$ on histone $\mathrm{H} 4$ bound by $\mathrm{TAF}_{\|} 250$ [21]. PTM targets can also reside on two distinct histone tails, such as H4K16ac and H3K4me3 bound by BPTF [7]. Dual recognition of histone PTMs is accomplished by tandem protein motifs within the histone-binding protein. Studies have shown that tandem motifs thermodynamically enhance binding affinity and specificity [22]. In order to compensate for the modest affinity of the CBX8 PCD [12] for its target, we added a second copy of H3K27me3-binding PCD to the N-terminus of PcTF to create $\mathrm{Pc}_{2} \mathrm{TF}$. Here, demonstrate that $\mathrm{Pc}_{2} \mathrm{TF}$ shows higher affinity for H3K27me3 in vitro. This activity corresponds with enhanced activation of a H3K27me3-repressed gene in cultured cells.

\section{RESULTS}

\section{Design of a bivalent synthetic chromatin-based transcriptional regulator}

We designed the $\mathrm{Pc}_{2} \mathrm{TF}$ protein to simultaneously recognize two copies of the histone posttranslational modification H3K27me3. The Polycomb chromodomain motif (PCD) consists of three $\beta$ strands packed against a C-terminal $\alpha$ helix, and a hydrophobic pocket formed by three aromatic residues that interact with a methyl-lysine sidechain [13,23] (Fig. 1A). The arrangement of histones within the nucleosome octamer suggests that $\mathrm{Pc}_{2} \mathrm{TF}$ might bind adjacent trimethylated H3K27 residues. A single nucleosome includes eight individual histone proteins. The central tetramer contains two copies of histone $\mathrm{H} 3$ and $\mathrm{H} 4$. The $\mathrm{H} 3$ proteins are oriented in cis so that the unfolded $\mathrm{N}$-terminal tails protrude away from the nucleosome in the same direction [24] (Fig. 1B). One or both $\mathrm{H} 3$ tails [25] can become trimethylated at lysine 27 by the enzyme enhancer of zeste $(\mathrm{EZH})$ [26]. Therefore, tandem PCDs in the multivalent protein $\mathrm{Pc}_{2} \mathrm{TF}$ might interact with two histone post translational modifications (PTMs) in a single nucleosome (Fig. 1B) or single PTMs on adjacent nucleosomes. 
A
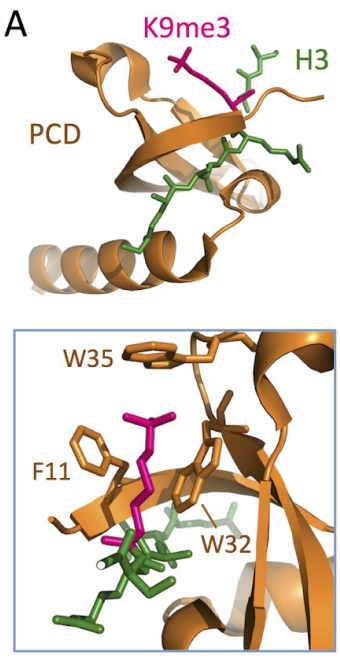

B

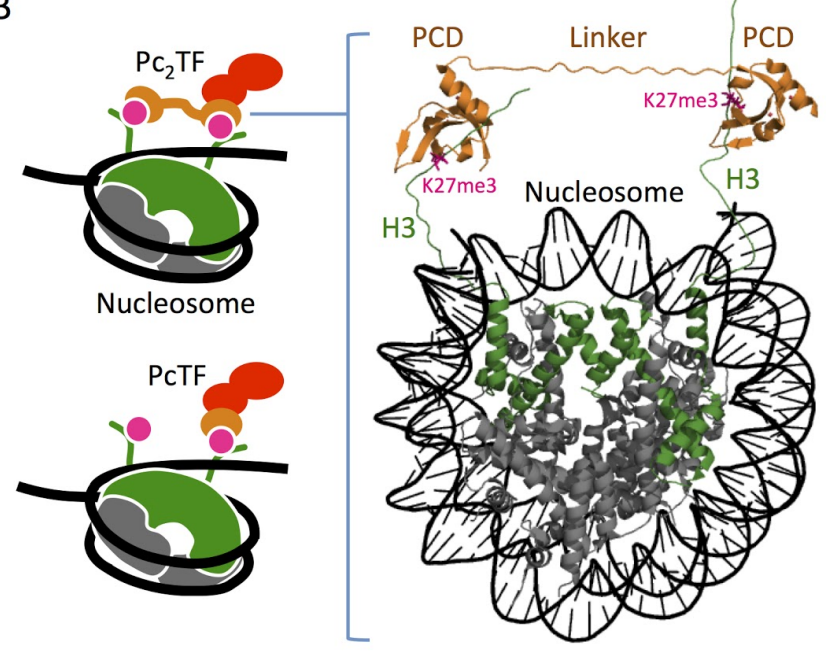

Figure 1. 3-D model layout to show the plausibility of $\mathrm{PC}_{2} T F$ binding to adjacent $\mathrm{H} 3 \mathrm{~K} 27$ me3 marks. (A) PCD (CBX8) in complex with trimethyl lysine (PDB 3191) [27]. Three residues form a hydrophobic cage and surround the Kme3 moiety (inset). (B) H3K27me3 recognition by synthetic fusion proteins that carry a single or tandem PCD domains (PCTF and $\mathrm{Pc}_{2} \mathrm{TF}$, respectively). The $3 \mathrm{D}$ rendering was composed in the PyMOL Molecular Graphics System, Version 1.3 Schrödinger, LLC (https://www.pymol.org/) using data for CBX8/H3K9me3 (PDB 4X3K) [28], and a whole nucleosome assembly (PDB 5AV8) [28,29] from the Protein Data Bank.

To identify a linker that allowed optimal binding with H3K27me3, we used an in vitro expression and ELISA procedure to test four $\mathrm{Pc}_{2}$ TF variants. Different lengths and physical characteristics were explored by using flexible glycine-serine linkers [30] and rigid alpha-helical $[31,32]$ linkers. Glycine and serine, amino acids with small side chains, have been used in a wide range protein engineering applications to build flexible linker peptides that have minimal interference with the function of tethered proteins [33]. It has been proposed that flexible, floppy linkers do not support the maximum distance between the tethered peptides [34]. Therefore, rigid linkers might perform better by stabilizing the distance between PCDs to support interactions with neighboring $\mathrm{K} 27$ me3 moieties. The $\mathrm{Pc}_{2}$ TF constructs included two tandem copies of the PCD separated by one of four linkers: flexible (GGGGS), long flexible (GGGGS), rigid (EAAAR), and long rigid (EAAAR) $)_{16}$. Based on a simplified layout of the interacting components (PCDs and a nucleosome carrying two H3K27me3 modifications) (Fig. 1B), we predicted that 20 amino acids would provide sufficient length for adjacent PCDs to bind simultaneously. The 80 amino acid linkers were used to determine the impact of increased spacing between PCDs.

Recombinant fusion proteins were produced using an efficient, cost-effective bacterial cell-free transcription translation (TXTL) system [35]. We used cell-free expression at the initial prototyping 
stage to eliminate time-consuming steps involved in E. coli over-expression and protein purification. Open reading frames for $\mathrm{Pc}_{2} \mathrm{TF}$ variants and a control protein with no binding domain $\left(\mathrm{Pc}_{\Delta} \mathrm{TF}\right)$ were cloned into a pET28 vector (Fig. 2A) and added into TXTL solution with a $\sigma 70-\mathrm{T} 7$ RNA Polymerase-expressing plasmid. Real-time detection of mCherry fluorescence in a Roche thermal cycler confirmed expression of recombinant proteins. For ELISA, TXTL products were added to microwells in which different C-terminal biotinylated histone peptides (H3 a.a. 21-44) were tethered via pre-coated neutravidin. Significant relative binding over background (unmodified K27 and K27ac) was detected for variants that contained the flexible (GGGGS), long flexible $(\text { GGGGS })_{16}$, and long rigid (EAAAR) ${ }_{16}$ linkers (Fig. 2C). The implications of these results are

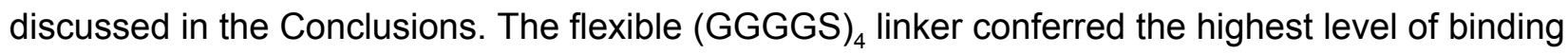
in this assay. Therefore, we used this variant in subsequent experiments to determine the impact of bivalency on the activity of synthetic, histone-binding effectors.
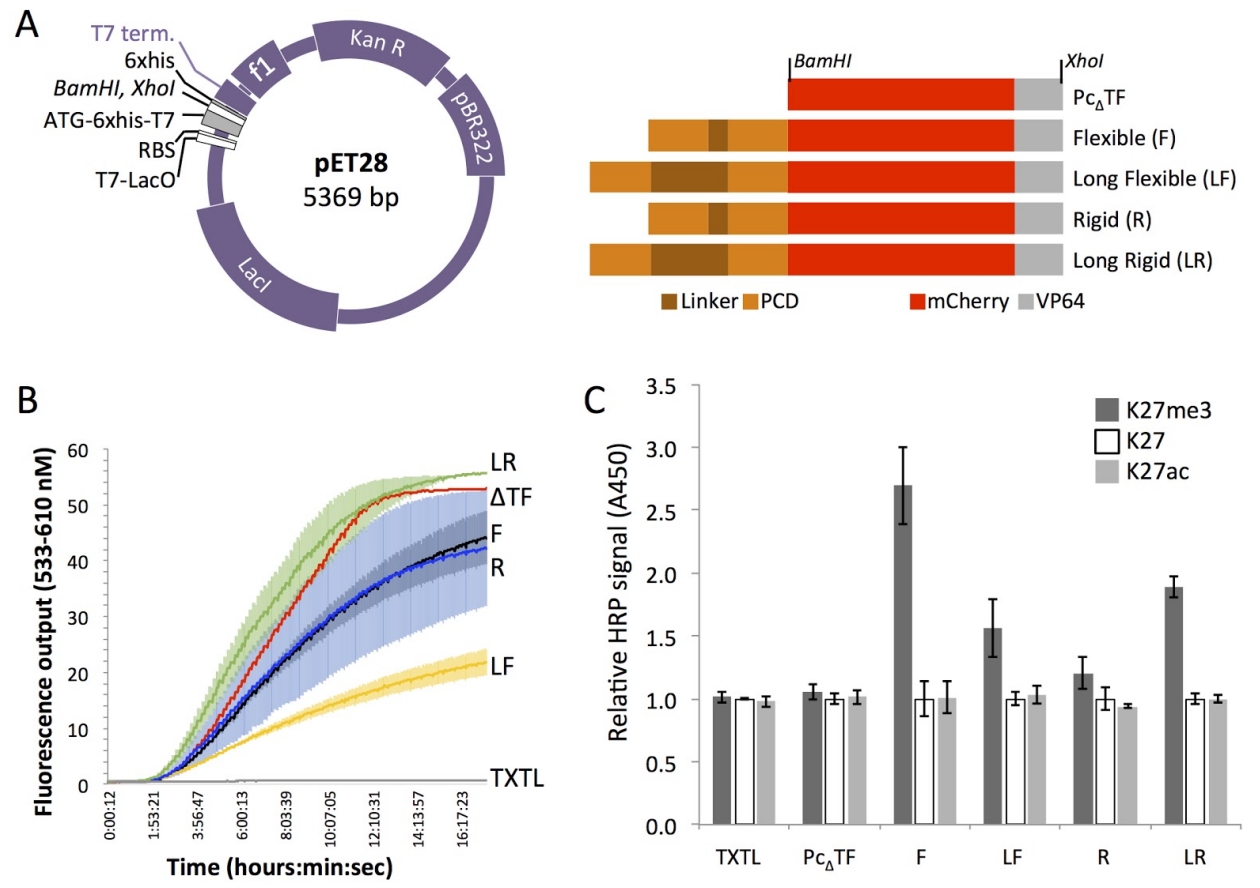

Figure 2. ELISA was used to determine binding affinity and specificity of $\mathrm{Pc}_{2} \mathrm{TF}$ variants that were expressed in a bacterial cell-free expression system. (A) Map of the expression vector and open reading frames (ORFs). Fusion-encoding ORFs were cloned in the pET28 vector at BamHI and Xhol. (B) Real-time detection of mCherry fluorescence was used to determine expression of recombinant protein in TXTL in a 96-well PCR plate in a Roche thermal cycler. $n=1$ for $\mathrm{Pc}_{\Delta} \mathrm{TF}$ and TXTL without DNA, $\mathrm{n}=3$ for others, shaded regions = SDM. (C) The bar chart shows binding of TXTL-expressed fusion proteins (or plasmid-free blank TXTL) with trimethyl-K27, unmodified, or off-target (acetyl-K27) histone H3 peptides. Values for each TXTL sample are normalized to unmodified $\mathrm{H} 3(\mathrm{n}=3$, bars $=\mathrm{SDM})$. 


\section{Bivalent $\mathrm{Pc}_{2}$ TF shows enhanced H3K27me3 binding compared to PcTF in vitro}

In ELISA experiments, the bivalent PCD fusion showed greater binding with H3K27me3 peptides compared to the single-PCD fusion protein. The Lacl-encoding pET28 vector (Fig. 2A) enabled IPTG-regulatable expression in E. coli, which was used to initiate high-yield production once the population reached log growth phase. Native polyacrylamide electrophoresis (PAGE) of lysates from IPTG-treated and untreated $E$. coli showed inducible production of the proteins at roughly the expected sizes: 37, 44, and 52 kilo Daltons for $\mathrm{Pc}_{\Delta} T F, \mathrm{PcTF}_{\text {, and }} \mathrm{Pc}_{2}$ TF respectively. Nickel-NTA column-purified proteins were soluble in $1 \mathrm{x}$ phosphate buffered saline (PBS). The visible red hue under white light, which is typical of the mCherry protein [36], indicated proper protein folding.

$50 \mathrm{nM}$ of purified PcTF, $\mathrm{Pc}_{2}$ TF, or $\mathrm{Pc}_{\Delta}$ TF was tested for interaction with different C-terminal biotinylated histone peptides as described for the TXTL-expressed proteins. PcTF and $\mathrm{Pc}_{2} \mathrm{TF}$ showed significant binding with H3K27me3 peptides compared to the control protein $\mathrm{Pc}_{\Delta} \mathrm{TF}$ (Fig. $3 \mathrm{C})$. Lack of binding with unmodified $\mathrm{H} 3$ or H3K27ac indicates that the interaction with $\mathrm{H} 3 \mathrm{~K} 27 \mathrm{me} 3$ is specific. $\mathrm{Pc}_{2}$ TF binding showed a 3-fold increase over PcTF. Therefore, at $50 \mathrm{nM}$ paired PCD motifs within the $\mathrm{Pc}_{2}$ TF protein confer significantly enhanced binding.

A

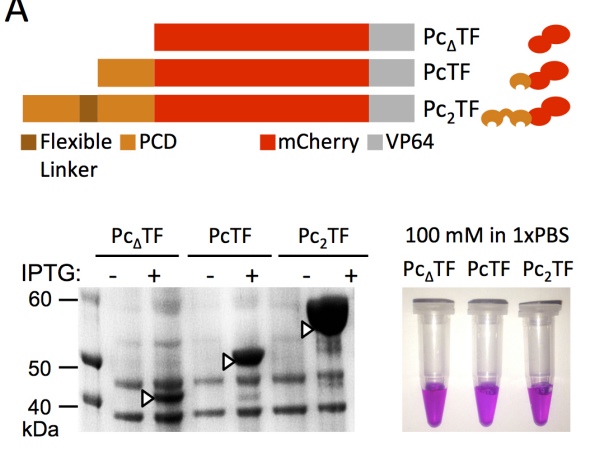

$$
\text { C }
$$

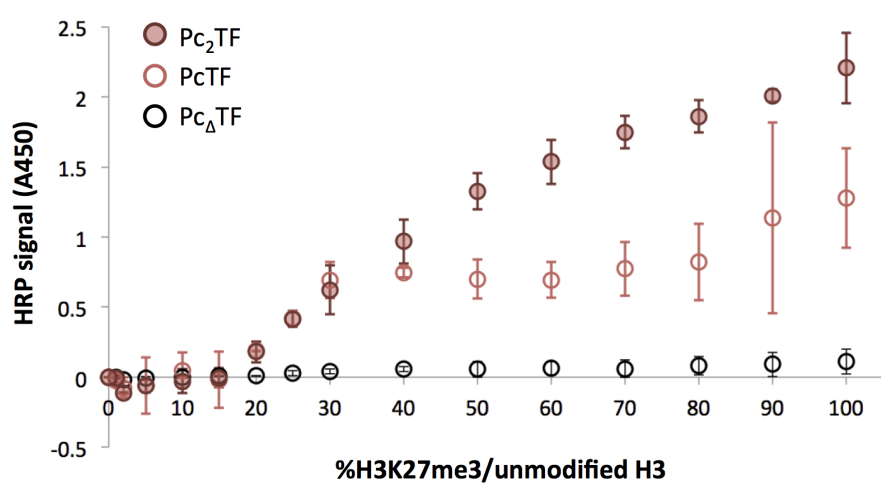

B

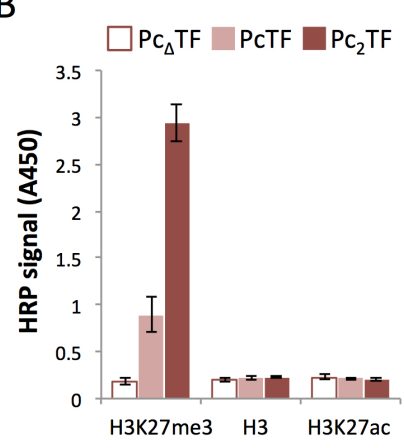

Figure 3. A bivalent PCD fusion peptide shows enhanced $\mathrm{H} 3 \mathrm{~K} 27 \mathrm{me} 3$ binding in vitro. (A) For high-yield expression, E. coli was transformed with pET28 plasmids encoding $\mathrm{Pc}_{\Delta} \mathrm{TF}$ (negative control), PcTF (single PCD), and the $\mathrm{Pc}_{2} \mathrm{TF}$ containing the flexible linker (GGGGS) . Native polyacrylamide gel electrophoresis (PAGE) of over-expressed proteins purified from E. coli. (C) ELISA was used to detect interaction of purified proteins with histone peptides that were trimethylated, unmodified, or acetylated at lysine 27 . The bar chart shows mean HRP signal $(n=4$ replicate ELISAs, bars $=$ SDM). 


\section{Bivalency contributes to Pc-transcription factor binding in an additive manner}

To determine whether the contribution of the additional PCD domain is additive or synergistic, we exposed tethered histone peptides to varying concentrations of soluble $\mathrm{PcTF}$ and $\mathrm{Pc}_{2} \mathrm{TF}$. Histone peptides were immobilized on glass slides as spot arrays by conjugation of biotinylated peptides to crosslinked neutravidin. Red fluorescent signal from the recombinant proteins allowed us to detect the amount of bound protein without the aid of a dye-conjugated antibody (Fig. 4A). We detected no interaction with unmodified histone $\mathrm{H} 3$ peptides and very little signal over background for the $\mathrm{Pc}_{\Delta}$ TF negative control. We carried out titrations of the recombinant proteins to determine affinities of each protein for 10, 20 and $50 \mu \mathrm{M}$ of tethered H3K27me3 ligand (Fig. S1). Figure 4B shows representative trials for $20 \mu \mathrm{M} \mathrm{H} 3 \mathrm{~K} 27$ me3. The calculated affinity of monovalent PcTF for $20 \mu \mathrm{M}$ of H3K27me3 was 5.14 - $8.95 \mu \mathrm{M}$ for four independent trials (Fig. 4B and S1). This range of values suggests similar or higher affinity of a fusion-PCD compared to isolated PCD peptides that were analyzed in other in vitro experiments. In other work, fluorescence polarization values for $\sim 60$ amino acid PCD homologues from Drosophila and mouse were $\mathrm{Kd}=5.0 \pm 1 \mu \mathrm{M}$ [13] and $165 \pm 20$ $\mu \mathrm{M}$ [12], respectively. Thus, $\mathrm{PCD}$ retains its intrinsic binding affinity as an $\mathrm{N}$-terminal motif within a fusion protein. Overall, at $10,20 \mu \mathrm{M}$ of ligand the $\mathrm{Kd}$ value of $\mathrm{Pc}_{2} \mathrm{TF}$ was roughly 2 -fold the value of PcTF (Fig. S1). The highest concentration of ligand showed a 4- to 6-fold increase for the bivalent protein in two trials (Fig. S1).

We can conservatively conclude that doubling of the N-terminal PCD motif increases affinity by 2 -fold for the majority the conditions in which we tested soluble proteins (ELISA and spot assay). This result suggests that the additional PCD motif contributes to protein binding in an additive manner in vitro. These results raise the question, what is the biological consequence of increased affinity in living cells where the distribution of H3K27me3 is much different? In the cellular chromatin environment, H3K27me3 can occur in cis on the radial surface of a single nucleosome (Fig. 1B), in trans where DNA bending brings the $\mathrm{H} 3$ tails of neighboring nucleosomes close together, or sparsely distributed across many nucleosomes. Furthermore, H3K27me3 marks in living cells are dynamic; the enzyme EZH1/2 adds methyl groups to H3K27, and KDM6A (UTX) and KDM6B (JMJD3) remove these marks (reviewed in [37]). Therefore, we set out to compare $\mathrm{Pc}_{2}$ TF to PcTF in a cell based system. 
A Arrayed $\mathrm{H} 3$ peptides

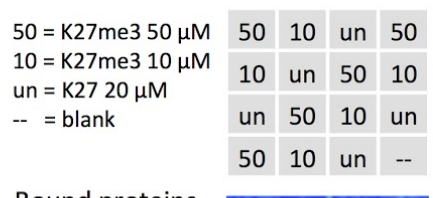

Bound proteins $(1.0 \mu \mathrm{M})$ :

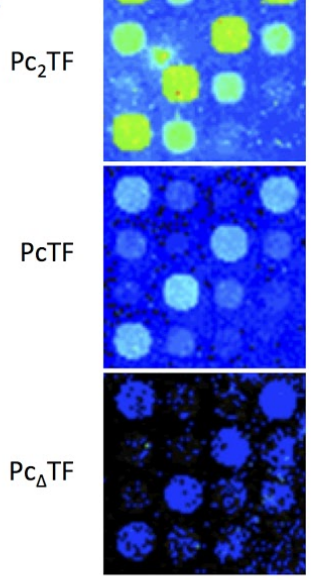

Binding: low
B $\quad$ K27me3
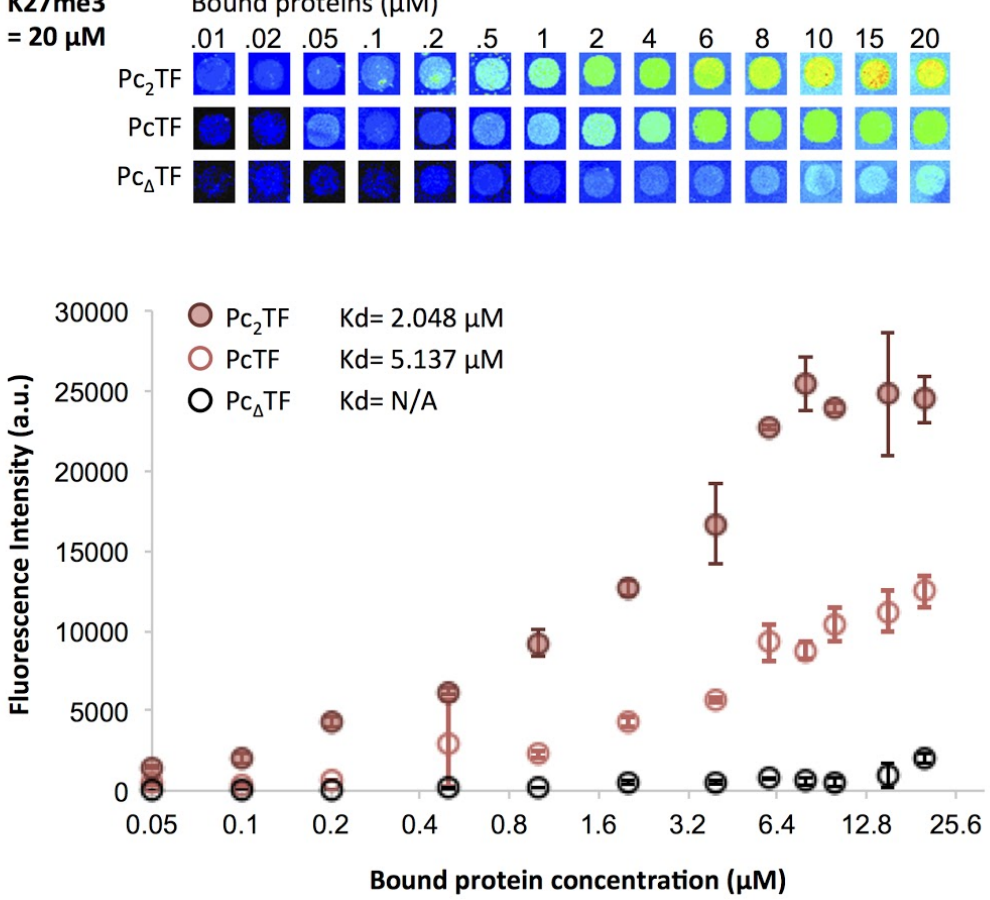

Figure 4. Binding affinities for $\mathrm{PcTF}$ and $\mathrm{Pc}_{2} \mathrm{TF}$ were determined using spot array assays. $(\mathrm{A})$ Slides were spotted with histone H3 peptides (K27me3 or unmodified K27) as depicted in the grid (top). (bottom) Pseudo-colored images of mCherry fluorescent signal probed with $1 \mu \mathrm{M}$ individual proteins. (B) Fluorescence signal versus concentration of protein added to the spot array was used to calculate binding affinity (Kd). The fluorescence and Kd values displayed in the graph are from a representative set of trials. For each trial $n=4$, bars $=$ SDM.

\section{Bivalent $\mathrm{Pc}_{2}$ TF activates a target gene in partially-silenced chromatin}

We determined the biological significance of enhanced affinity by comparing the gene-regulation activities of $\mathrm{Pc}_{2}$ TF and PcTF at an epigenetically silenced Tk-luciferase reporter gene in HEK293 cells. To compare the activities of the fusion proteins in live cells, we cloned the open reading frames for PcTF and $\mathrm{PC}_{2}$ TF into a mammalian expression vector called MV10 (Fig. 5A). We observed nuclear RFP signals 48 hours after transfection with Lipofectamine-plasmid DNA complexes (Fig. 5B) and used RFP-positive cell frequency (Table S2) to normalize gene activation in subsequent assays.

We used a H3K27me3-enriched firefly luciferase reporter gene to compare transcriptional regulation activities of the methyl-histone-binding fusion proteins. HEK293 Gal4-EED/luc cells are engineered to enable doxycycline-mediated control of chromatin state at a chromosomally integrated firefly luciferase transgene [38] (Fig. 5C). We and others have demonstrated that doxycycline-induced expression of the Gal4-EED fusion protein leads to accumulation of 
H3K27me3 near the Gal4 UAS operator and silencing of Tk-luciferase expression [10,38,39]. Prior to transfection with fusion protein-expressing plasmids, cells were cultured in $1 \mathrm{ug} / \mathrm{mL}$ dox to induce Gal4-EED-mediated silencing at Tk-luciferase for 48 or 96 hours, then grown without dox for 48 hours to allow for loss of Gal4-EED from the promoter region. Tk-luciferase repression reaches steady state at 96 hours point [39], and repression is maintained by epigenetic inheritance after loss of Gal4-EED [38].

We transfected untreated or dox-treated cells with $\mathrm{Pc}_{\Delta} \mathrm{TF}, \mathrm{PcTF}$, or $\mathrm{Pc}_{2}$ TF. Cells were collected for luciferase activity assays 48 hours after transfection. $\mathrm{PC}_{\Delta} \mathrm{TF}$ did not stimulate activation of Tk-luciferase above the negative control background. In cells where silencing was induced for 96 hours, $\mathrm{Pc}_{2}$ TF showed roughly twofold activation of Tk-luciferase compared to PcTF. This twofold relative enhancement of gene regulation activity is consistent with the in vitro assays (ELISA and spot arrays). Therefore, the relative binding and affinity observed for purified proteins translates to gene regulation activity in live cells at the model reporter gene tested here.

We also found that $\mathrm{Pc}_{2}$ TF activates Tk-luciferase prior to dox-induced silencing. Previously, we demonstrated that untreated Gal4-EED/luc cells show an intermediate, partially silenced level of Tk-luciferase expression [39] compared to fully active Tk-luciferase in a "Luc14" parental cell line that lacks the Gal4-EED gene [39]. Furthermore, H3K27me3 was detected via ChIP-qPCR near the luciferase promoter $(T k)$ in uninduced Gal4-EED/luc cells at significantly higher levels than in Luc14 cells. Dox treatment resulted in a further decrease in Tk-luciferase expression and a significant increase in $\mathrm{H} 3 \mathrm{~K} 27$ me3 accumulation. In the experiments reported here, basal Tk-luciferase expression (Fig. 5D) agrees with independent experiments from our previously reported study (0.02 - 0.07 luciferase activity per cell, a.u.) [39]. The uninduced state may have low levels of H3K27me3 at nucleosomes near the reporter gene in all cells, or high levels of $\mathrm{H} 3 \mathrm{~K} 27 \mathrm{me} 3$ at the reporter gene in a small proportion of cells in the population. In contrast to $\mathrm{Pc}_{2} \mathrm{TF}_{\text {, }}$ monovalent PcTF only activated Tk-luciferase after silenced chromatin had been induced for 96 hours. These results suggest that $\mathrm{PC}_{2}$ TF is more tolerant of low levels of $\mathrm{H} 3 \mathrm{~K} 27 \mathrm{me} 3$ in cellular chromatin. In the Conclusions section, we discuss how this idea is consistent with the behavior of the natural bivalent chromatin complex Rpd3S. 
A

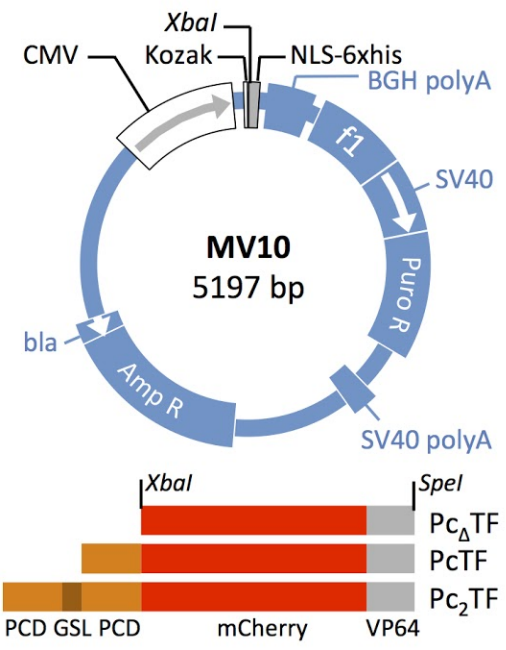

C

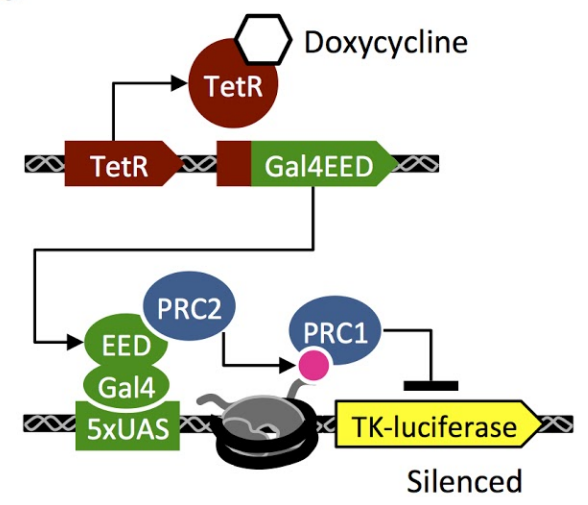

B
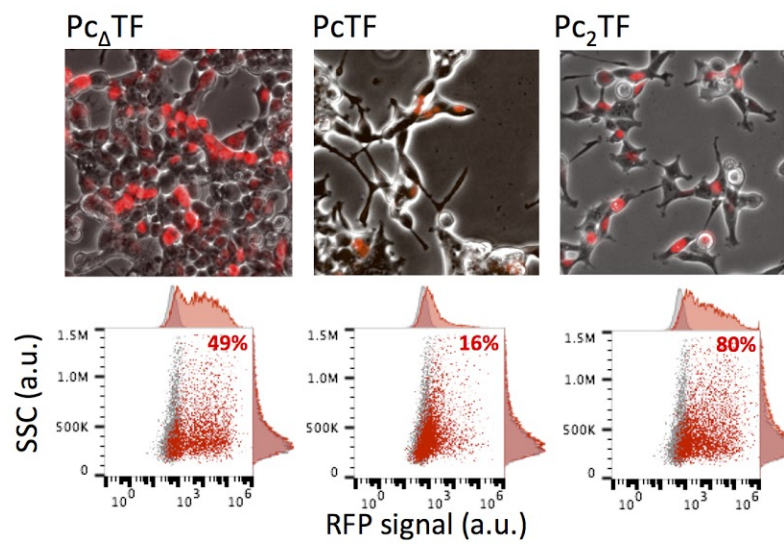

$\square$ No plasmid

Transfected

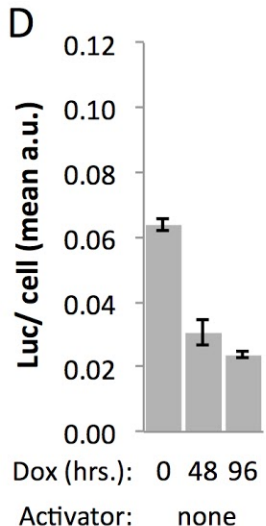

Figure 5. $\mathrm{Pc}_{2}$ TF stimulates expression at a Polycomb-silenced reporter gene. (A) Fusion constructs were cloned into the MV10 vector at Xbal. (B) Fluorescence microscopy confirms nuclear localization of the fusion proteins. Representative examples are shown here. Expression frequency (\%RFP-positive live cells) was determined by flow cytometry. Data for dox untreated cells are shown. See Table S2 for all values. (C) An engineered HEK293 cell line, Gal4-EED/luc, was used for doxycycline-mediated control of H3K27me3 and Polycomb-mediated silencing at a Tk-luciferase reporter. (D) (left) Tk-luciferase expression determined by firefly luciferase assays ( $n=3$, bars $=S D M)$. Expression is partially silenced prior to dox treatment, as demonstrated previously [39] and becomes fully repressed at 96 hours. Response of Polycomb-silenced Tk-luciferase to $\mathrm{Pc}_{\Delta} \mathrm{TF}, \mathrm{PcTF}$, and $\mathrm{Pc}_{2} \mathrm{TF}$ is shown in the right bar chart (RFP = RFP-positive cell frequency; $\mathrm{n}$ = 3, each scaled to mean $\mathrm{Pc}_{\Delta} \mathrm{TF}$ luc/cell/RFP; bars = SDM).

\section{CONCLUSIONS}

In our previous work, we have demonstrated the use of a monovalent synthetic effector to activate chromatin-silenced genes in live cells. Natural bivalent chromatin proteins that recognize two histone post translational modifications at once suggest a broader design space for synthetic 
chromatin effectors. Our application of bivalency to design a synthetic fusion protein produced three important advances for engineering synthetic chromatin effectors. First, we determined that synthetic linkers allow tethered histone PTM-binding peptides to function within the context of a fusion protein in vitro and in live cells. Second, we have established that tandem PCDs increase affinity and gene regulation activity by roughly 2 -fold compared to a single PCD. Finally, we demonstrated that tolerance for low target PTM levels can be reproduced in a synthetic bivalent effector.

Here, we demonstrated that different synthetic linkers allow tethered histone PTM-binding peptides to bind in vitro to varying degrees. We observed weaker binding for the longer flexible linker (80 amino acids) compared to the shorter linker in our ELISA experiment. This result is likely due to lower production of the long flexible linker variant in TXTL. Given that both variants showed binding above background, GGGGS-repeat number may not significantly affect bivalent PCD engagement with H3K27me3 in vitro. For the rigid linker-tethered PCDs, only the longer length (80 amino acids) appeared to support binding. Assuming that this variant protein was properly folded, lack of binding over background for the shorter EAAAR-repeat variant could be caused by suboptimal rotation, i.e. in trans instead of in cis, of the second PCD away from the 2-D binding surface in the ELISA well. Indeed, alpha-helical linkers have been used to control rotational orientation in pairs of tethered histone binding domains [7] and Zinc Finger DNA binding domains [32]. In the context of cellular chromatin where looping and folding occurs, H3K27me3 would not necessarily be constrained to one face of the $\mathrm{Pc}_{2}$ TF protein. Valuable lessons and perhaps greater $\mathrm{Pc}_{2}$ TF performance might be acquired by exploring additional linker variants in cells as well as in vitro. Such work is beyond the scope of the studies reported here, which accomplished a major step by identifying a functional bivalent synthetic effector protein that specifically interacts with its target H3K27me3.

We have established that tandem PCDs increase affinity in vitro and gene regulation activity in live cells by roughly 2-fold compared to a monovalent PCD. This is the first report of intramolecular, synthetic bivalency for a gene-silencing-associated mark (H3K27me3). Prior to our report, studies of multivalent engagement with histones have exclusively focused naturally-occurring, tandem histone-binding motifs [40-42]. The wide distribution of multivalency within bromodomain family [6] and other effector proteins [19] suggests that multivalent engagement has an important, evolutionarily-conserved biological role. Multivalency appears to largely be represented by cell-cycle and gene-activating effectors. Relatively few multivalent proteins that recognize silencing marks have been studied in biophysical detail. Examples include 
the chromodomains of the Arabidopsis protein CMT3 [43] and the mammalian protein HP1 $\beta$ [44]; as bivalent homodimers, these proteins show enhanced interaction with their respective ligands $\mathrm{H} 3 \mathrm{~K} 9$ meK27me and $\mathrm{H} 3 \mathrm{~K} 9 \mathrm{me} 3$. $\mathrm{Pc}_{2}$ TF is novel in its composition of histone-binding motifs: adjacent, identical Polycomb chromodomains within a single peptide. Therefore, its activity in vitro and in cells provides new insights into the recognition of histone marks by effector proteins.

In the context of cellular chromatin, $\mathrm{Pc}_{2}$ TF-mediated activation of a target gene (Tk-luciferase) appears to tolerate low levels of $\mathrm{H} 3 \mathrm{~K} 27 \mathrm{me}$, whereas the monovalent variant PcTF required greater accumulation of the mark in order to activate the same target. Our ChIP mapping data [39] confirm that low levels appear at the ectopic H3K27me3 site (Tk-luciferase) on average. The limited resolution of ChIP-qPCR on chromatin extracted from a large cell population only allows us to surmise that either a few cells have a high local concentration of H3K27me3 near the Tk promoter, or that many cells have sparse H3K27me3 in the same location. Enhanced affinity might allow $\mathrm{Pc}_{2}$ TF to bind more stably (i.e., have a reduced off-rate) at low $\mathrm{H} 3 \mathrm{~K} 27 \mathrm{me} 3$ levels and therefore stimulate Tk-luciferase expression at higher frequencies. Additionally, the tandem PCD domains might allow stable docking in regions of sparse H3K27me3. Such is the case for the purported bivalent chromatin modifier complex RpdS3, which contains the H3K36me-binding chromodomain protein Eaf3 and an additional histone-tail-binding motif, the PHD finger of Rco1. RpdS3 binds with very similar affinities to nucleosome pairs (dinucleosomes) in which the H3K36me mark is located on both or just one of the nucleosomes [45]. Direct recognition of H3K36me by the Rco1 PHD has not yet been established, and weak interactions with the histone globular domain are thought to contribute to RpdS3 binding; it is unclear whether RpdS3 is 'truly' bivalent. Our observation of $\mathrm{Pc}_{2}$ TF binding with histone tail peptides in the absence of whole nucleosomes suggests that PTM depletion tolerance in general does not require interactions with histone core regions.

Further engineering efforts to achieve greater, nonlinear enhancement of $\mathrm{PcTF} / \mathrm{Pc}_{2} \mathrm{TF}$ may require changes within the PCD binding motif. The hydrophobic interaction between the methylammonium cage and the methyl-lysine moiety (Fig. 1A) depends upon proper positioning of PCD residues that appear discontinuously in the primary sequence; this positioning requires specific intramolecular contacts of peptide residues within the PCD fold. Reverse engineering and de novo design of a new binding pocket through randomization of sequences would likely yield many non-functional proteins. K27-adjacent interactions that support that contribute to interaction with the histone tail $[13,23]$ could be leveraged to enhance affinity. However, increasing the stability by introducing addition hydrogen bonding could overwhelm the hydrophobic, K27me3-specific 
interaction, and allow PCD to recognize unmodified tails or off-target modifications. Trade-offs between affinity and specificity pose formidable challenges to enhancing PCD affinity. Therefore, the most practical strategy for identifying alternative PCDs is to leverage H3K27me3-specific orthologs and paralogs from various species [46]. It will be important to determine cross-reactivity with various histone modifications since CBX PCD peptides have been shown to bind H3K9me3 [12].

Multivalent engagement of combinatorial histone marks has recently become a key line of evidence to support the controversial histone code hypothesis. Rationally-designed synthetic multivalency will advance this important area of research by exploring functions beyond the limits of pre-existing natural multivalent proteins. Furthermore, engineered chromatin effectors provide a practical tool to support artificial regulation of gene expression states through direct engagement with highly conserved components of chromatin, i.e. histone tails and their modifications. Therapeutic, synthetic gene regulators that leverage this mechanism could help circumvent the shortcomings of epigenetic inhibitors, which target chromatin enzymes that are mutated in some cancers [47]. In conclusion, our findings demonstrate that synthetic biology is a powerful tool for fundamental investigations of chromatin biology and epigenetic engineering.

\section{MATERIALS AND METHODS}

\section{Plasmid Constructs for TXTL and Bacterial Expression}

Constructs (Fig. 2A, Fig. 3A) were assembled as BioBrick compatible fragments in vector V0120 [48]. Fragments were PCR-amplified with Phusion polymerase using primers 1-6 (Table S1) and a

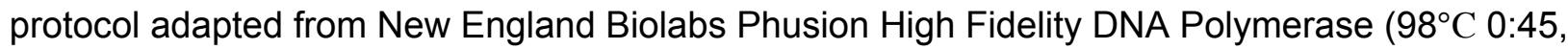

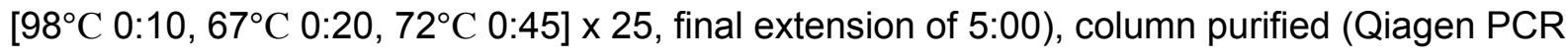
Cleanup Kit), and double-digested with BamHI and Xhol (Thermo Fisher FastDigest). BamHI/Xhol-digested inserts and 50 - 75 ng BamHI/Xhol linearized pET28(+) vector were ligated at a 3:1 molar ratio in a $20 \mu \mathrm{L}$ reaction as described in the New England Biolabs (NEB) protocol for T4 ligase (M0202). $5 \mu \mathrm{L}$ of each ligation was incubated with $50 \mu \mathrm{L}$ Turbo competent DH5-alpha $E$. coli (NEB) on ice for 5 minutes, transferred to $45^{\circ} \mathrm{C}$ for 45 seconds, then to ice for 5 minutes, and allowed to recover in $350 \mu \mathrm{L} \mathrm{SOC}$ at $37^{\circ} \mathrm{C}$ with shaking for 30 minutes. Pelleted cells were resuspended in $50 \mathrm{uL} \mathrm{SOC}$, plated on LB agar (50 ug/mL kanamycin), and grown at $37^{\circ} \mathrm{C}$ overnight. Colony PCR was performed to identify positive ligation results using primers 6 and 7 (Table S1) and the GoTaq Promega protocol. Plasmids were cloned, extracted (Sigma GeneElute 
Plasmid Miniprep Kit), and Sanger sequenced for verification prior to protein expression in cell-free TXTL or in E. coli. Annotated sequences for all pET28 constructs are available online at Benchling-Hayneslab: Synthetic Chromatin Actuators 2.0 (https://benchling.com/hayneslab/f/rmSYkAAU-synthetic-chromatin-actuators-2-0).

\section{TXTL: Cell-free Expression}

TXTL reactions were set up with the following conditions as previously described [35]: $9 \mu \mathrm{L}$ lysate, $10 \mathrm{nM}$ final template vector, $0.5 \mathrm{nM} \sigma 70-\mathrm{T} 7 \mathrm{RNA}$ pol vector to a total of $12 \mu \mathrm{L}$. A Roche Lightcycler 480 was used to detect mCherry fluorescence with the following: protocol: $30^{\circ} \mathrm{C}$ for 10 minutes, bring to $29^{\circ} \mathrm{C}$ for 1 second, scan $533-610 \mathrm{~nm}$, repeat 96 times (total $16 \mathrm{hrs}$ ).

\section{E. coli Expression and Purification of Proteins}

All selection media contained 50ug/mL kanamycin. $\mathrm{Pc}_{\Delta} \mathrm{TF}, \mathrm{PcTF}$, and $\mathrm{Pc}_{2} \mathrm{TF}$ in $\mathrm{pET} 28$ were transformed into Rosetta 2pLys DE3 cells and plated on LB agar and grown at $37^{\circ} \mathrm{C}$ overnight. The next day, a single colony from each was used to inoculate $50 \mathrm{~mL} \mathrm{LB}$ and grown overnight at $37^{\circ} \mathrm{C}$ at 300 RPM. The next day, 1 liter of LB in a baffled Erlenmeyer flask was inoculated to an OD600 of 0.1 . The cultures were grown to an OD600 $=0.6$, induced with IPTG ( $1 \mathrm{mM}$ final concentration) and allowed to express $\mathrm{PC}_{\Delta}$ TF and PcTF at $37^{\circ} \mathrm{C}$ for 5 hours with shaking (220 RPM). $\mathrm{PC}_{2}$ TF-expression was carried out overnight at room temperature with shaking (220 RPM) to aid solubility of the protein. Cell disruption and protein purification are described in detail in Supplemental Materials and Methods.

\section{ELISA Assays}

All steps were carried out at room temperature except specifically noted, and all incubations and washes were agitated at 800 RPM on an Eppendorf Thermomixer R. Clear bottom plates (Greiner bio-one \#655101) were coated in $50 \mathrm{uL}$ of $20 \mathrm{ng} / \mu \mathrm{L}$ neutravidin in PBS pH. 8.0 overnight at $4 \mathrm{C}$. The plates were washed the next day $3 x$ with $200 \mu \mathrm{L} 0.2 \%$ PBST with 5 minutes of shaking at 800 RPM between washes. The plate was blocked for $30 \mathrm{~min}$ at $800 \mathrm{RPM}$ at room temperature with 200 uL 5\% BSA in PBST followed by $3 x$ washes of 200 uL $0.2 \%$ PBST for 5 minutes each at 800 RPM. $50 \mu \mathrm{L}$ of $1 \mathrm{uM}$ biotinylated peptides (Anaspec) in $0.2 \%$ PBST were incubated at room temperature for 1 hour at 800 RPM, followed by $3 x$ washes of $200 \mu \mathrm{L} 0.2 \%$ PBST for 5 minutes at 800 RPM. The plate was then blocked for 30 minutes with $200 \mu \mathrm{L} 5 \%$ skim milk in $0.2 \%$ PBST at room temperature shaking at 800 RPM. 50 uL of purified proteins (put optimal concentration here) 
diluted in 5\% skim milk in PBST were incubated for 1 hour at room temperature at 800 RPM. The wells were washed $3 x$ with $200 \mu \mathrm{L} 5 \%$ skim milk in PBST with 5 minutes of 800 RPM shaking. 100 $\mu \mathrm{L}$ of $1: 3000$ chicken anti-mcherry (company and product number) in $5 \%$ skim milk in $0.2 \%$ PBST were incubated for 1 hour at 800 RPM and room temperature followed by 2x of $200 \mathrm{uL} 5 \%$ skim milk in $0.2 \%$ PBST for 5 min each at 800 RPM. 100 uL of 1:3000 Rabbit anti chicken HRP (RCYHRP Genetel $0.5 \mathrm{mg} / \mathrm{mL}$ ) in $5 \%$ skim milk in $0.2 \%$ PBST was incubated for 30 minutes at room temperature and 800 RPM. The plate was washed $5 x$ with $200 \mu \mathrm{L} 0.2 \%$ PBST for three minutes each at 800 RPM. The plate was incubated with $100 \mu \mathrm{L}$ of 1-step Ultra TMB-ELISA (Thermo-Fisher \#34029) for 15 minutes while blocked from light. The plate was then deactivated with $100 \mu \mathrm{L} 2.0 \mathrm{M}$ sulfuric acid, incubated for 2 minutes, and read at $450 \mathrm{~nm}$.

\section{Peptide Spot Arrays}

APTES functionalized glass slides were coated with $200 \mu \mathrm{L}$ of $1: 1$ (v/v) $40 \mathrm{mg} / \mathrm{mL}$ BS3 crosslinking solution and $1 \mathrm{mg} / \mathrm{mL}$ neutravidin with a cover slide and incubated overnight at $4^{\circ} \mathrm{C}$. The next day, the cover slide was removed and the slide was rinsed $3 x$ with $0.2 \%$ PBST for 5 minutes each. The slides were deactivated by incubation with $\mathrm{Na}_{2} \mathrm{CO}_{3} / \mathrm{NaHCO}_{3}$ buffer $\mathrm{pH} 9.4$ for 30 minutes. The slides were quickly rinsed with $\mathrm{ddH}_{2} \mathrm{O}$, and centrifuged to dry at $1200 \mathrm{RPM}$ for 2 minutes. The slides were printed with biotinylated peptides at concentrations of 10,20 , or $50 \mu \mathrm{M}$ in $20 \%$ glycerol and PBS with a pin-printer and incubated at room temperature for $1 \mathrm{~h}$ to allow the biotinylated peptide bind to neutravidin. The distance between spots were 600 um. The slide was rinsed with $\mathrm{ddH}_{2} \mathrm{O}$ as described above and blocked with superblock for 1 hour at room temperature. Proteins were diluted in superblock and incubated on the slide for 1 hour at room temperature. The slides were rinsed with $0.2 \%$ PBST for 3 minutes each followed by quick rinsing with ddH2O $3 x$ and centrifuged dry (as described). Red fluorescent protein (mCherry) signal was detected at $50 \%$ gain and $50 \%$ intensity on a PowerScanner at $635 \mathrm{~nm}$ and $535 \mathrm{~nm}, 10$ um resolution. The slides were also scanned at $75 \%-75 \%$ and $100 \%-100 \%$ to to obtain a sutiable signal to noise ratio. Arraypro software was used to quantify the median intensity values for each spot and background levels. Graphpad Prism software was used to fit the data to the binding saturation nonlinear regression equation $y=\left(B_{\max }{ }^{*} X\right) /\left(K_{d}+x\right)$, where $B_{\max }$ is the highest binding value and $X$ is the concentration of protein.

\section{Plasmid Constructs for Mammalian Expression}


MV10 was constructed from pcDNA3.1(+) (Invitrogen) with the following modifications. The CMV promoter was removed via Spel digestion and T4 ligase recircularization. A dsDNA fragment that encodes Kozak (ribosome binding site), Xbal, a nuclear localization sequence, 6xhistidine, and a stop codon

(5'-cccgccgccaccatggagtctagacccaagaaaaagcgcaaggtacaccatcaccaccatcacgcgtaaagctgag) with Spel overhangs at both ends (ctag/t) was inserted at Xbal. CMV (Spel/Xbal fragment) was reintroduced upstream of Kozak at Spel. Proper orientation of inserts was confirmed by Sanger sequencing. Constructs PcTF and $\mathrm{Pc}_{2}$ TF (Fig. 5A) were PCR-amplified (Phusion) with primers 9 and 10 (Table S1), double-digested with Xbal and Spel, and column-purified (Qiagen PCR Purification, 28104). Construct $\mathrm{PC}_{\Delta}$ TF (Fig. 5A) was double-digested with Xbal and Spel (Thermo Fisher FastDigest) and isolated by electrophoresis and gel purification. Xbal/Spel fragments and $25 \mathrm{ng}$ Xbal-linearized, dephosphorylated MV10 vector were ligated at a 2:1 molar ratio in a $10 \mu \mathrm{L}$ reaction as described in the Roche protocol for the Rapid DNA Ligation Kit (11635379001 Roche), using $1.0 \mu \mathrm{L}$ NEB T4 ligase instead of the supplied enzyme. All $10 \mu \mathrm{L}$ of each ligation was incubated with $50 \mu \mathrm{L}$ Turbo competent DH5-alpha E. coli (New England Biolabs) on ice for 5 minutes, transferred to $45^{\circ} \mathrm{C}$ for 45 seconds, then to ice for 5 minutes. Cells were plated directly on pre-warmed LB agar (100 ug/mL ampicillin) without recovery and grown at $37^{\circ} \mathrm{C}$ overnight. Plasmid DNA was prepared (Sigma GeneElute Plasmid Miniprep Kit) from $5 \mathrm{~mL}$ cultures inoculated with single colonies. Proper orientation of the inserts was determined by Xbal and Pstl double-digestion of prepped plasmids and Sanger sequencing. Annotated sequences for all MV10 constructs are available online at Benchling-Hayneslab: Synthetic Chromatin Actuators 2.0 (https://benchling.com/hayneslab/f_rmSYkAAU-synthetic-chromatin-actuators-2-0).

\section{Cell Culture and Transfection}

HEK293-Gal4-EED cells were grown in Dulbecco's modified Eagle's medium (DMEM) supplemented with $10 \%$ tetracycline-free fetal bovine serum and $1 \%$ penicillin and streptomycin at $37^{\circ} \mathrm{C}$ in a humidified $\mathrm{CO}_{2}$ incubator. Silencing of the reporter gene (Tk-luciferase) was induced by supplementing the media with $1 \mu \mathrm{g} / \mathrm{mL}$ of dox for 48 or 96 hours. For wash-out of doxycycline, growth medium was removed and replaced with dox-minus medium supplemented with $0.5 \mu \mathrm{g} / \mathrm{mL}$ puromycin to select for the transgenic anti-Gal4-EED shRNA [38], and grown for 5 days. Prior to transfection, dox treated or untreated cells were plated in 12-well culture dishes at $40 \%$ confluency ( $1.0 \mathrm{E} 5$ cells per well) in $2 \mathrm{~mL}$ pen/strep-free growth medium. Transient transfections were carried 
out by adding $100 \mu \mathrm{L}$ of DNA/Lipofectamine complexes to each well: $1 \mu \mathrm{g}$ DNA (10 $\mu \mathrm{L}), 3 \mu \mathrm{L}$ Lipofectamine LTX (Invitrogen), $87 \mu \mathrm{L}$ Opti-MEM.

\section{Imaging and Flow Cytometry}

48 hours after transfection, cellular mCherry (580/610 excitation/emission) was imaged in culture dishes on a Nikon Eclipse Ti wide field fluorescent microscope (MEA53100, filter G-2E/C). For flow cytometry, the growth medium was removed, adherent cells were gently washed with 1xPBS, harvested by trypsinization (Trypsin-EDTA $0.25 \%$, Gibco), resuspended in growth medium to neutralize the trypsin, pelleted at $200 \mathrm{RCF}$, and resuspended in 1xPBS supplemented with $1 \%$ FBS. Red fluorescent signal from mCherry was detected on a BD Accuri C6 flow cytometer (675 nm LP filter) using CFlow Plus software. Data were further processed using FlowJo 10.0. RFP values for transfected cells are listed in Table S2.

\section{Luciferase Assays}

For the same samples that were used for flow cytometry, cells per $100 \mu \mathrm{L}$ were determined by flow cytometry (BD Accuri C6). $100 \mu \mathrm{L}$ of cells or 1 xPBS (blank) were incubated with $100 \mu \mathrm{L}$ of complete luciferase assay reagent as described in the protocol for the Biotium Firefly Luciferase Assay Kit (89138-960) and in previous work [39] in Corning and Costar 96-well Cell Culture Plates, black, clear bottom (Bioexpress). Chemiluminescence was detected using a Synergy $\mathrm{H} 1$ Multi-Mode Reader (Biotek). Luciferase expression per cell was calculated as follows: Sample Luciferase per cell $=$ [Sample Luciferase signal] -1 XPBS blank signal/[cell count $\times(100 \mu \mathrm{L} / 20 \mu \mathrm{L})]$. Values for transfected cells were divided by RFP frequency (Table S2).

\section{ACKNOWLEDGEMENTS}

The work was supported by the $\mathrm{NIH} \mathrm{NCl} \mathrm{(K01} \mathrm{CA188164} \mathrm{to} \mathrm{K.A.H.).} \mathrm{S.T.,} \mathrm{D.V.,} \mathrm{and} \mathrm{L.S.} \mathrm{were}$ supported by the Biological Design PhD Program at ASU. D.V. was also supported by the Western Alliance to Expand Student Opportunities (NSF HRD 1401190). We thank undergraduate researchers B. Laughlin, C. Gardner, D. Tze, and J. Xu for early contributions to plasmid construction. We also thank V. Noireaux for his generous gift of TXTL reagents.

\section{CONTRIBUTIONS}


S.T. built bacterial expression constructs, produced purified protein, performed protein validation, and executed the TXTL experiments. S.T. and L.S. designed and executed ELISA and spot array analyses. D.V. built mammalian expression constructs, and performed transfections, flow cytometry and imaging, dox-mediated silencing, and luciferase activity assays. K.A.H. was responsible for oversight of the experiments and writing of the manuscript.

\section{REFERENCES}

1. Tekel S, Haynes KA. Molecular Structures Guide the Engineering of Chromatin. Nucleic Acids Res. 2017; in press.

2. Cano-Rodriguez D, Rots MG. Epigenetic Editing: On the Verge of Reprogramming Gene Expression at Will. Curr Genet Med Rep. 2016;4: 170-179.

3. Park M, Keung AJ, Khalil AS. The epigenome: the next substrate for engineering. Genome Biol. 2016;17: 183.

4. Platero JS, Hartnett T, Eissenberg JC. Functional analysis of the chromo domain of HP1. EMBO J. 1995;14: 3977-3986.

5. Messmer S, Franke A, Paro R. Analysis of the functional role of the Polycomb chromo domain in Drosophila melanogaster. Genes Dev. 1992;6: 1241-1254.

6. Filippakopoulos P, Picaud S, Mangos M, Keates T, Lambert J-P, Barsyte-Lovejoy D, et al. Histone recognition and large-scale structural analysis of the human bromodomain family. Cell. 2012;149: 214-231.

7. Ruthenburg AJ, Li H, Milne TA, Dewell S, McGinty RK, Yuen M, et al. Recognition of a mononucleosomal histone modification pattern by BPTF via multivalent interactions. Cell. 2011;145: 692-706.

8. Gatchalian J, Fütterer A, Rothbart SB, Tong Q, Rincon-Arano H, Sánchez de Diego A, et al. Dido3 PHD modulates cell differentiation and division. Cell Rep. 2013;4: 148-158.

9. Wang R, You J. Mechanistic analysis of the role of bromodomain-containing protein 4 (BRD4) in BRD4-NUT oncoprotein-induced transcriptional activation. J Biol Chem. 2015;290: 2744-2758.

10. Haynes KA, Silver PA. Synthetic reversal of epigenetic silencing. J Biol Chem. 2011;286: 27176-27182.

11. Nyer DB, Daer RM, Vargas D, Hom C, Haynes KA. Regulation of cancer epigenomes with a histone-binding synthetic transcription factor. npj Genomic Med. 2017;2: 1074.

12. Bernstein E, Duncan EM, Masui O, Gil J, Heard E, Allis CD. Mouse polycomb proteins bind differentially to methylated histone $\mathrm{H} 3$ and RNA and are enriched in facultative heterochromatin. Mol Cell Biol. 2006;26: 2560-2569. 
13. Fischle W, Wang Y, Jacobs SA, Kim Y, Allis CD, Khorasanizadeh S. Molecular basis for the discrimination of repressive methyl-lysine marks in histone $\mathrm{H} 3$ by Polycomb and HP1 chromodomains. Genes Dev. 2003;17: 1870-1881.

14. Meckler JF, Bhakta MS, Kim M-S, Ovadia R, Habrian CH, Zykovich A, et al. Quantitative analysis of TALE-DNA interactions suggests polarity effects. Nucleic Acids Res. 2013;41: 4118-4128.

15. Jantz D, Berg JM. Probing the DNA-binding affinity and specificity of designed zinc finger proteins. Biophys J. 2010;98: 852-860.

16. Greisman HA, Pabo CO. A general strategy for selecting high-affinity zinc finger proteins for diverse DNA target sites. Science. 1997;275: 657-661.

17. Sternberg SH, Redding S, Jinek M, Greene EC, Doudna JA. DNA interrogation by the CRISPR RNA-guided endonuclease Cas9. Nature. 2014;507: 62-67.

18. Haynes KA, Silver PA. Synthetic reversal of epigenetic silencing. J Biol Chem. 2011;286: 27176-27182.

19. Ruthenburg AJ, Li H, Patel DJ, Allis CD. Multivalent engagement of chromatin modifications by linked binding modules. Nat Rev Mol Cell Biol. 2007;8: 983-994.

20. Su X, Zhu G, Ding X, Lee SY, Dou Y, Zhu B, et al. Molecular basis underlying histone H3 lysine-arginine methylation pattern readout by Spin/Ssty repeats of Spindlin1. Genes Dev. 2014;28: 622-636.

21. Jacobson RH. Structure and Function of a Human TAFII250 Double Bromodomain Module. Science. 2000;288: 1422-1425.

22. Voigt $P$, Reinberg $D$. Histone tails: ideal motifs for probing epigenetics through chemical biology approaches. Chembiochem. 2011;12: 236-252.

23. Min JR, Zhang Y, Xu R-M. Structural Basis for specific binding of polycomb chromodomain to histone H3 methylated at K27 [Internet]. 2003. doi:10.2210/pdb1pfb/pdb

24. Luger K, Mäder AW, Richmond RK, Sargent DF, Richmond TJ. Crystal structure of the nucleosome core particle at 2.8 A resolution. Nature. 1997;389: 251-260.

25. Voigt P, LeRoy G, Drury WJ 3rd, Zee BM, Son J, Beck DB, et al. Asymmetrically modified nucleosomes. Cell. 2012;151: 181-193.

26. Kuzmichev A, Nishioka K, Erdjument-Bromage H, Tempst P, Reinberg D. Histone methyltransferase activity associated with a human multiprotein complex containing the Enhancer of Zeste protein. Genes Dev. 2002;16: 2893-2905.

27. Kaustov L, Ouyang H, Amaya M, Lemak A, Nady N, Duan S, et al. Recognition and specificity determinants of the human cbx chromodomains. J Biol Chem. 2011;286: 521-529.

28. Ren C, Morohashi K, Plotnikov AN, Jakoncic J, Smith SG, Li J, et al. Small-molecule modulators of methyl-lysine binding for the CBX7 chromodomain. Chem Biol. 2015;22: 
$161-168$.

29. Wakamori M, Fujii Y, Suka N, Shirouzu M, Sakamoto K, Umehara T, et al. Intra- and inter-nucleosomal interactions of the histone $\mathrm{H} 4$ tail revealed with a human nucleosome core particle with genetically-incorporated H4 tetra-acetylation. Sci Rep. 2015;5: 17204.

30. Wriggers W, Chakravarty S, Jennings PA. Control of protein functional dynamics by peptide linkers. Biopolymers. 2005;80: 736-746.

31. Merutka G, Shalongo W, Stellwagen E. A model peptide with enhanced helicity. Biochemistry. 1991;30: 4245-4248.

32. Yan W, Imanishi M, Futaki S, Sugiura Y. a-Helical Linker of an Artificial 6-Zinc Finger Peptide Contributes to Selective DNA Binding to a Discontinuous Recognition Sequence†.

Biochemistry. 2007;46: 8517-8524.

33. Chen X, Zaro JL, Shen W-C. Fusion protein linkers: property, design and functionality. Adv Drug Deliv Rev. 2013;65: 1357-1369.

34. Klein JS, Jiang S, Galimidi RP, Keeffe JR, Bjorkman PJ. Design and characterization of structured protein linkers with differing flexibilities. Protein Eng Des Sel. 2014;27: 325-330.

35. Caschera F, Noireaux V. Synthesis of $2.3 \mathrm{mg} / \mathrm{ml}$ of protein with an all Escherichia coli cell-free transcription-translation system. Biochimie. 2014;99: 162-168.

36. Shen Y, Chen Y, Wu J, Shaner NC, Campbell RE. Engineering of mCherry variants with long Stokes shift, red-shifted fluorescence, and low cytotoxicity. PLoS One. 2017;12: e0171257.

37. Swigut T, Wysocka J. H3K27 demethylases, at long last. Cell. 2007;131: 29-32.

38. Hansen KH, Bracken AP, Pasini D, Dietrich N, Gehani SS, Monrad A, et al. A model for transmission of the H3K27me3 epigenetic mark. Nat Cell Biol. 2008;10: 1291-1300.

39. Daer RM, Cutts JP, Brafman DA, Haynes KA. The Impact of Chromatin Dynamics on Cas9-Mediated Genome Editing in Human Cells. ACS Synth Biol. 2017;6: 428-438.

40. Su X, Zhu G, Ding X, Lee SY, Dou Y, Zhu B, et al. Molecular basis underlying histone H3 lysine-arginine methylation pattern readout by Spin/Ssty repeats of Spindlin1. Genes Dev. 2014;28: 622-636.

41. Jacobson RH. Structure and Function of a Human TAFII250 Double Bromodomain Module. Science. 2000;288: 1422-1425.

42. Ruthenburg AJ, Li H, Milne TA, Dewell S, McGinty RK, Yuen M, et al. Recognition of a mononucleosomal histone modification pattern by BPTF via multivalent interactions. Cell. 2011;145: 692-706.

43. Lindroth AM, Shultis D, Jasencakova Z, Fuchs J, Johnson L, Schubert D, et al. Dual histone $\mathrm{H} 3$ methylation marks at lysines 9 and 27 required for interaction with CHROMOMETHYLASE3. EMBO J. 2004;23: 4286-4296.

44. Hiragami-Hamada K, Soeroes S, Nikolov M, Wilkins B, Kreuz S, Chen C, et al. Dynamic and 
flexible H3K9me3 bridging via HP1 $\beta$ dimerization establishes a plastic state of condensed chromatin. Nat Commun. 2016;7: 11310.

45. Huh J-W, Wu J, Lee C-H, Yun M, Gilada D, Brautigam CA, et al. Multivalent di-nucleosome recognition enables the Rpd3S histone deacetylase complex to tolerate decreased H3K36 methylation levels. EMBO J. 2012;31: 3564-3574.

46. Senthilkumar R, Mishra RK. Novel motifs distinguish multiple homologues of Polycomb in vertebrates: expansion and diversification of the epigenetic toolkit. BMC Genomics. 2009;10: 549.

47. Gibaja V, Shen F, Harari J, Korn J, Ruddy D, Saenz-Vash V, et al. Development of secondary mutations in wild-type and mutant EZH2 alleles cooperates to confer resistance to $\mathrm{EZH} 2$ inhibitors. Oncogene. 2016;35: 558-566.

48. Phillips I, Silver P. A New Biobrick Assembly Strategy Designed for Facile Protein Engineering. 2006; Available: http://hdl.handle.net/1721.1/32535 